\title{
Hypersonic swizzle sticks: jets, fossil cavities and turbulence in molecular clouds
}

\author{
Andrew J Cunningham ${ }^{1}$, Adam Frank ${ }^{1}$, Eric G Blackman ${ }^{1}$ \\ and Alice Quillen ${ }^{1}$
}

${ }^{1}$ Department of Physics and Astronomy, University of Rochester, Rochester, NY 14627, USA email: ajc4@pas.rochester.edu

\begin{abstract}
The ubiquity and high density of outflows from young stars in clusters make them an intriguing candidate for the source of turbulence energy in molecular clouds. In this contribution we discuss new studies, both observational and theoretical, which address the issue of jet/outflow interactions and their ability to drive turbulent flows in molecular clouds. Our results are surprising in that they show that fossil cavities, rather than bow shocks from active outflows, constitute the mechanism of re-energizing turbulence. We first present simulations which show that collisions between active jets are ineffective at converting directed momentum and energy in outflows into turbulence. This effect comes from the ability of radiative cooling to constrain the surface area through which colliding outflows entrain ambient gas. We next discuss observational results which demonstrate that fossil cavities from "extinct" outflows are abundant in molecular material surrounding clusters such as NGC 1333. These structures, rather than the bow shocks of active outflows, comprise the missing link between outflow energy input and re-energizing turbulence. In a separate theoretical/simulation study we confirm that the evolution of cavities from decaying outflow sources leads to structures which match the observations of fossil cavities. Finally we present new results of outflow propagation in a fully turbulent medium exploring the explicit mechanisms for the transfer of energy and momentum between the driving wind and the turbulent environment.
\end{abstract}

Keywords. ISM: clouds,ISM: jets and outflows

\section{Introduction}

Star formation occurs within Molecular Clouds (MCs), complex structures whose physical evolution is still not clearly understood (Ballesteros-Paredes et al. (2006)). MCs are hierarchical structures with smaller substructures known as clumps and cores. Star formation is believed to occur in cores with larger clusters forming from more massive cores. The expected lifetimes for molecular clouds has become a topic of considerable debate as numerical simulations have shown that MHD turbulence, the nominal means of support for clouds against self-gravity, decays on a crossing timescale (Goldreich \& Kwan (1974), Arons \& Max (1975), Stone et al. (1998), Mac Low \& Klessen (2004)). In light of this result the traditional view that MCs are long-lived, quasi-static equilibrium structures has been challenged by a paradigm in which star formation occurs on a timescale comparable to the free-fall time (Ballesteros-Paredes et al. (1999), Elmegreen (2000), Hartmann(2003)). In the former case turbulence in the cloud is an important source of support and regulation of the star formation efficiency and it must be resupplied over time. In the latter case turbulence is produced with the cloud (Yamaguchi et al. (2001)) or only needs to be driven up to the point that a cloud is disrupted.

Feedback from protostars forming within a MC has been cited by many authors as a principle means of either re-energizing turbulence or disrupting clouds (Bally \& 

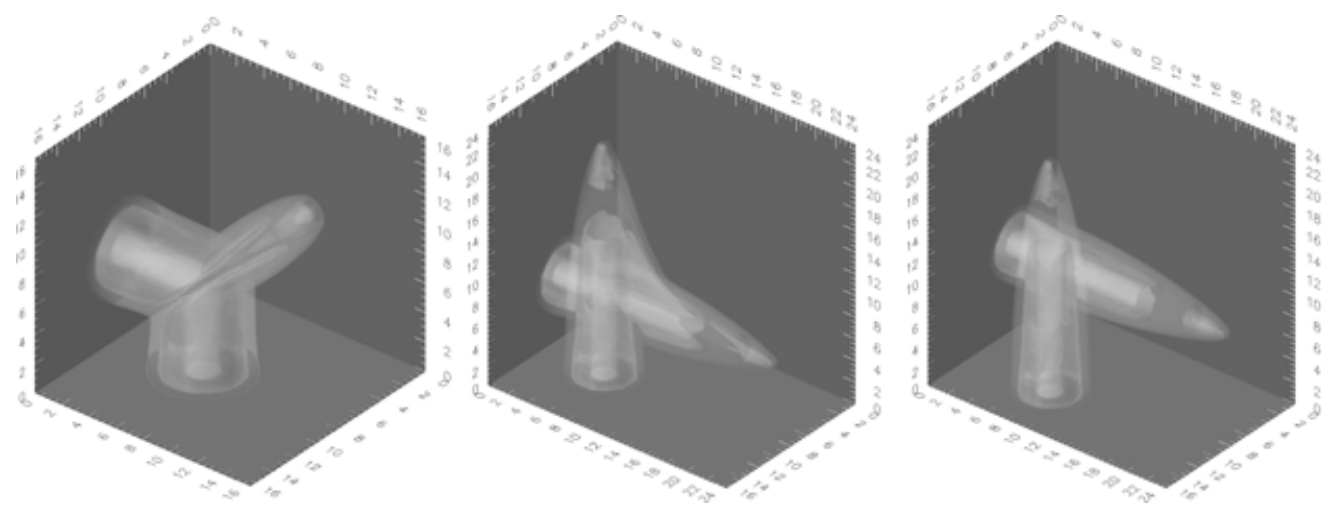

Figure 1. Volume rendered image of density from AMR simulations of two jets undergoing a $90^{\circ}$ collision displaced by an impact parameters of 0,1 jet radius, and non-interacting from left to right.

Reipurth (2001)). When massive stars form their ionization fronts, strong stellar winds and eventual supernova blastwaves are expected to be the major contributor to feedback (Krumholz \& McKee (2005)). In lower mass clusters and environments where the effects of massive stars have not been felt protostellar outflows will likely be the dominant form of feedback. In these cases, even if energy is re-supplied from supra-cluster scales, at some wavenumber the outflow injection may come to dominate global dynamics. In fact, energetic outflows associated with low and moderate mass young stellar objects are known to exert a strong effect on their parent molecular clouds (for a recent review see Bally et al. (2006)). Young stellar outflows in settings such as NGC 1333 have been shown to contain sufficient kinetic energy to excite a significant fraction of supersonic turbulence in their surrounds and/or unbind and disperse portions of their parent cloud. (Bally \& Reipurth (2001), Knee \& Sandell (2000), Warin et al. (1996)).

In this contribution we explore the role of outflows in returning energy and momentum to the surrounding media from young stars. We focus first on simulation results exploring the role of collisions between active outflows, demonstrating that these types of interaction are not more effective than non-interacting outflows at setting the ambient material in motion or generating randomized isotropic motions which are characteristic of turbulence. Recognizing that the driving properties of star formation outflows (in particular mass loss rates) evolve on timescales shorter than the duration of the outflow, we then focus on fossil outflows first from an observational viewpoint and then in terms of simulations designed to recover and interpret those observations.

\section{Active outflow collisions}

We estimate $N_{\text {critical }}$, the protostellar density that achieves a volume fill ratio of $10 \%$ bowshock overlap. Above this density expect the effect of collisions between outflows to become appreciable. Assuming typical values for the protostellar outflow size, bow shock radius, outflow lifetime and cloud lifetime we find $N_{\text {critical }}=500 \mathrm{pc}^{-3}$ (Cunningham et al. (2006A)). This is comparable to the protostellar density of many star forming regions. Outflow interactions are therefore statistically likely to occur in a typical star forming region.

Motivated by the implied likelihood for jet collisions, we explored the efficacy of active outflow interactions in stirring the ambient medium via 3-D AMR simulations of jet collisions (Cunningham et al. (2006B)). Our study focused on hydrodynamic simulations 

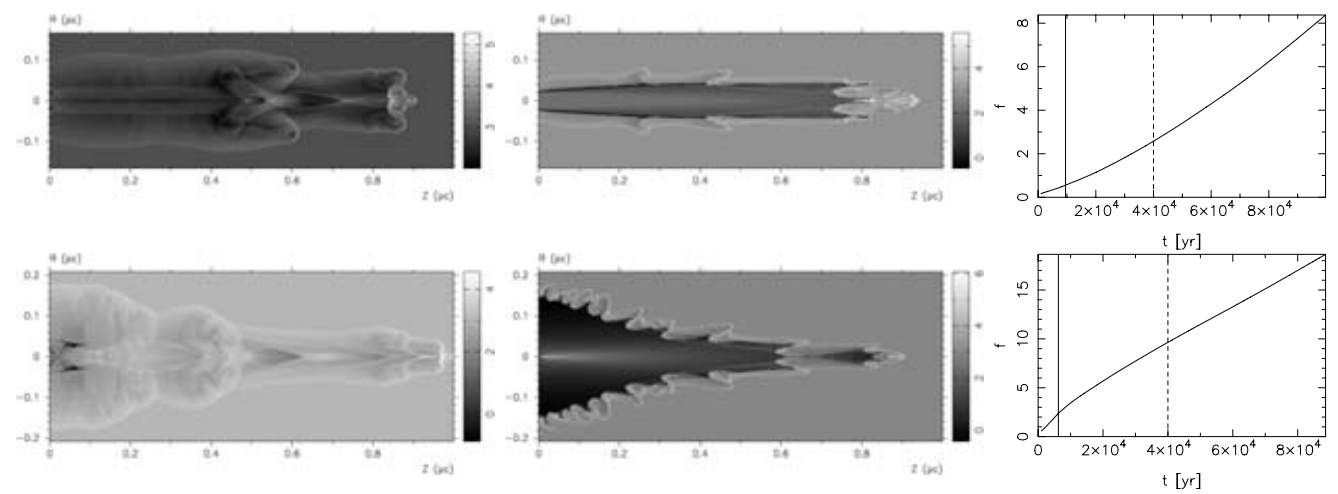

Figure 2. Left: Simulations of fossil cavities driven by winds which are active for $10^{4} \mathrm{yr}$. Number density images are shown at $\sim 10^{5}$ yr. Note that backfilling via rarefaction waves has occurred. Center: Outflows driven by active winds shown for comparison with frame taken at $\sim 3 x 104 \mathrm{yr}$. Right: fractional difference between the approximate scaling relation of Quillen et al. (2005) and the simulation results. Between the two vertical lines delineating the wind shut-off time and the time at which the flows have decelerated to $\sim 1 \mathrm{kms}^{-1}$, the scaling relation and simulation differ by a factor of order unity. The top panel shows the results for a collimated jet driven flow and the lower panel for a wide angle wind driven flow.

of the interaction of two orthogonal outflows at several impact parameters (figure 1). The simulations included the effect of radiative energy loss on the flow and we investigated the role of the impact parameter and degrees of collimation. The simulations were carried out in 3D using the AstroBEAR adaptive mesh refinement (AMR) code.

Surprisingly, our results indicated that the high degrees of compression of outflow material, achieved through radiative shocks near the vertex of the interaction, prevent the redirected outflow from spraying over a large spatial region. Furthermore, the collision reduces the redirected outflow's ability to entrain and impart momentum into the ambient cloud. We note the study by Li \& Nakamura (2006) explored outflow interactions with a collapsing turbulent cloud and concluded that outflow activity could re-energize turbulence. These studies do not contradict our results as we agree with their main conclusion however, their simulations were of quite low resolution ( $128^{3}$ for the entire cluster) and could not adequately resolve the interactions of individual outflows or include the outflow power evolution (a topic we will explore in the next section). Thus combining the results of our simulations with consideration of the probabilities of outflow collisions led us to conclude that individual low velocity fossil outflows, interacting on long timescales, provide the principle coupling between outflows and the cloud.

\section{Fossil outflows}

Many authors have relied on "0-D" estimates of the energy present in active outflows and compare these with cloud turbulent energy (Bally \& Reipurth (2001)). Studies of individual objects however express a more complicated picture. In particular the explicit time-dependent nature of the coupling between cloud material and outflows is not addressed in these estimates. Outflow power evolves rapidly in time in the strongest phases (Class 0 sources) lasting a fraction of the star formation timescale. Thus outflows will continually be turning on and fading across the history of an active star forming region.

The pitfalls of ignoring the temporal domain was highlighted in a recent study of NGC1333 (Quillen et al. (2005)). In this work it was found that velocity dispersions, measured in $13 \mathrm{CO}$, did not vary across the cloud. There was no link between active 

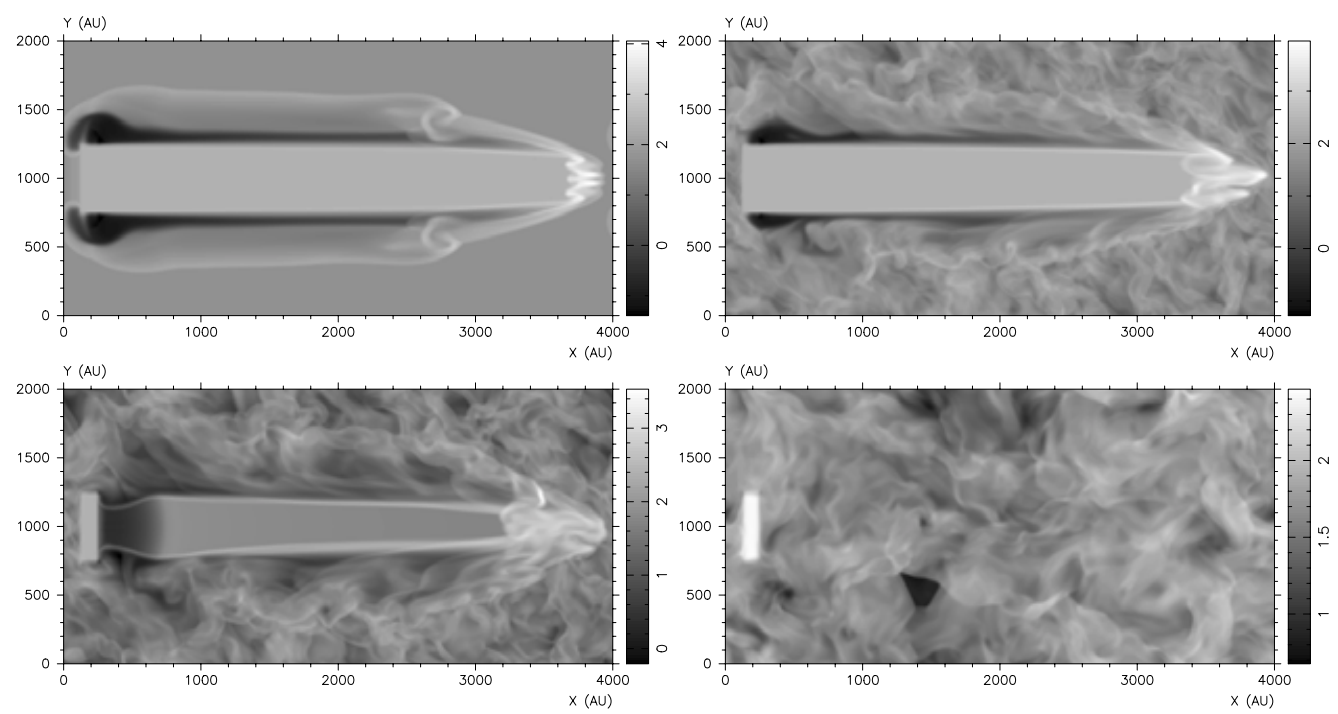

Figure 3. 2-D number density slices of 3-D simulations of jets with different decay times driven into turbulent media. Top Left: Control in which constant jet driven into quiescent media. Top Right: Constant jet driven into turbulent media. Lower Left: Slowly decaying jet driven into turbulent media. Lower Right: Short pulsed jet driven into turbulent media. Note the effect of the turbulent media on then bow shock. In short pulse jet simulations, the outflowing material has been completely subsumed by the turbulence. This is the expected behavior for long-extinct outflows.

outflows and turbulence. Instead a new class of outflow signature was identified in the form of fossil cavities. These fossil cavities proved to be a smoking gun showing strong coupling between outflows and the molecular cloud. Cavities at a range of sizes and velocities were observed in the cloud. Twenty cavities were identified with typical diameter of about $0.1-0.2 \mathrm{pc}$, and velocity widths $1-3 \mathrm{kms}^{-1}$. If these cavities were simply empty regions in the cloud, the timescale for them to fill in would be less than a million years implying that they were created relatively recently.

In a recent, more detailed study, AMR simulations of cavity evolution using full $\mathrm{H}_{2}$ chemistry and cooling (Cunningham et al. (2006B)) were performed. In this work jets and wide angle winds were simulated with an injected momentum flux that decreased in time (figure 2). These simulations were compared with runs with constant momentum flux. The decaying flux models exhibited deceleration of the outflow head and backfilling via expansion off of the cavity walls. They also recovered observed morphological signatures including lower density contrasts in comparison to the continuously driven counterparts. Most important, the simulations agree with the scaling relations developed by Quillen et al. (2005) to estimate cavity momentum from their readily observable properties (figure 2, right). This work also provided synthetic observations in terms of P-V diagrams which demonstrate that fossil cavities form both jets and wide angle outflows are characterized by linear "Hubble-law" expansions patterns superimposed on "spur" patterns indicative of the head of a bow shock. These should prove useful in future observational work.

\section{Conclusion \& future work}

We have shown that fossil cavities, rather than active outflows, may be the direct link between stellar injection of mechanical energy and turbulence within young clusters. 
Thus while turbulent energy may be supplied at larger scales to the clouds as a whole (via supernova or gravitational collapse) which then cascades down, there is also a separate injection of energy at smaller scales which constitutes a feedback from the stars within the cluster. Future work will need to make the interplay between turbulence and energy injection from jets more explicit. Figure 3 shows initial work in this direction in the form of simulations of jets into fully turbulent media and the subsequent evolution of the jet driven cavity (Cunningham et al. (2007)). By the time several turbulent crossing times have elapsed, the cavities have slowed via the entrainment of the ambient turbulent gas sufficiently to be subsumed into and become indistinguishable from the turbulent motions of the cloud. Through this process, the jet momentum will act to feed the turbulence that will ultimately destroy it.

\section{References}

Arons, J. \& Max, C. E. 1975, ApJ 196, L77

Ballesteros-Paredes, J., Hartmann, L. \& Vázquez-Semadeni, E. 1999, ApJ 527, 285

Ballesteros-Paredes, J., Klessen, R., Mac Low, M-M. \& Vazquez-Semadeni, E, 2006, in: B. Reipurth, D. Jewitt, \& K. Keil (eds.), Protostars and Planets V (Tucson: Univ. of Arizona), in press

Bally, J., Reipurth, B. \& Davis, C., 2006, in: B. Reipurth, D. Jewitt, \& K. Keil (eds.), Protostars and Planets $V$ (Tucson: Univ. of Arizona), in press

Bally, J. \& Reipurth, B. 2001, ARA\&A 39, 403

Cunningham, A. J., Frank, A. \& Blackman, E. G. 2006A, ApJ 646, 1059

Cunningham, A. J., Frank, A., Blackman, E. G. \& Quillen, A., 2006B, ApJ in press

Cunningham, A. J., Frank, A., Blackman, E. G. \& Quillen, A., 2007, in prep

Elmegreen, B. G. 2000, ApJ 530, 277

Goldreich, P. \& Kwan, J. 1974, ApJ 189, 441

Hartmann, L. 2003, ApJ 585, 398

Knee, L. B. G. \& Sandell, G. 2000, $A \& A$ A 361, 671

Krumholz, M. R. \& McKee, C. F. 2005, ApJ 630, 250

Li, Z.-Y. \& Nakamura, F. 2006, ApJ 640, L187

Quillen, A. C., Thorndike, S. L., Cunningham, A., Frank, A., Gutermuth, R. A., Blackman, E. G., Pipher, J. L. \& Ridge, N. 2005, ApJ 632, 941

Mac Low, M.-M. \& Klessen, R. S. 2004, Rev. Mod. Phys. 76, 125

Stone, J. M., Ostriker, E. C. \& Gammie, C. F. 1998, ApJ 508, L99

Warin, S., Castets, A., Langer, W. D., Wilson, R. W. \& Pagani, L. 1996, A $\& A$ A 306, 935

Yamaguchi, R., et al. 2001, PASJ 53, 985 